\title{
Evidence for mast cells contributing to neuromuscular pathology in an inherited model of ALS
}

\author{
Emiliano Trias, ${ }^{1}$ Sofía Ibarburu, ${ }^{1}$ Romina Barreto-Núñez, ${ }^{1}$ Valentina Varela, ${ }^{1}$ Ivan C. Moura, $, 3,3,4,5,6,7$ \\ Patrice Dubreuil, ${ }^{7,8,9}$ Olivier Hermine, ${ }^{2,3,4,5,6,7,8,10,11}$ Joseph S. Beckman, ${ }^{12}$ and Luis Barbeito ${ }^{1}$ \\ IInstitut Pasteur de Montevideo, Uruguay. ${ }^{2}$ Imagine Institute, Hôpital Necker, Paris, France. ${ }^{3}$ INSERM UMR 1163, \\ Laboratory of Cellular and Molecular Mechanisms of Hematological Disorders and Therapeutic Implications, Paris, \\ France. ${ }^{4}$ Paris Descartes-Sorbonne Paris Cité University, Imagine Institute, Paris, France. ${ }^{5}$ CNRS ERL 8254, Paris, France. \\ ${ }^{6}$ Laboratory of Excellence GR-Ex, Paris, France. ' ${ }^{2}$ quipe Labélisée par la Ligue Nationale contre le cancer, Parisa, France. \\ ${ }^{8} \mathrm{AB}$ Science, Paris, France. ${ }^{9}$ Signaling, Hematopoiesis and Mechanism of Oncogenesis, Cancer Research Center of \\ Marseille (CRCM), Inserm U1068, Institut Paoli-Calmettes, Aix-Marseille University UM105, CNRS UMR7258, Marseille, \\ France. ${ }^{10}$ Department of Hematology, Necker Hospital, Paris, France. "Centre national de référence des mastocytoses \\ (CEREMAST), Paris, France. ${ }^{12}$ Linus Pauling Institute, Department of Biochemistry and Biophysics, Environmental Health \\ Sciences Center, Oregon State University, Corvallis, Oregon, USA
}

Evidence indicates that neuroinflammation contributes to motor neuron degeneration in amyotrophic lateral sclerosis (ALS), a fatal neurodegenerative disease leading to progressive muscular paralysis. However, it remains elusive whether inflammatory cells can interact with degenerating distal motor axons, influencing the progressive denervation of neuromuscular junctions (NMJs). By analyzing the muscle extensor digitorum longus (EDL) following paralysis onset in the $\mathrm{SOD}^{\mathrm{C} 93 \mathrm{~A}}$ rat model, we have observed a massive infiltration and degranulation of mast cells, starting after paralysis onset and correlating with progressive NMJ denervation. Remarkably, mast cells accumulated around degenerating motor axons and NMJs, and were also associated with macrophages. Mast cell accumulation and degranulation in paralytic EDL muscle was prevented by systemic treatment over 15 days with masitinib, a tyrosine kinase inhibitor currently in clinical trials for ALS exhibiting pharmacological activity affecting mast cells and microglia. Masitinib-induced mast cell reduction resulted in a $35 \%$ decrease in NMJ denervation and reduced motor deficits as compared with vehicle-treated rats. Masitinib also normalized macrophage infiltration, as well as regressive changes in Schwann cells and capillary networks observed in advanced paralysis. These findings provide evidence for mast cell contribution to distal axonopathy and paralysis progression in ALS, a mechanism that can be therapeutically targeted by masitinib.

Conflict of interest: $\mathrm{OH}$ and $\mathrm{PD}$ are cofounders and shareholders of $\mathrm{AB}$ Science.

Submitted: June 27, 2017 Accepted: September 11, 2017 Published: October 19, 2017

Reference information: JCI Insight. 2017;2(20):e95934. https://doi.org/10.1172/jici. insight.95934.

\section{Introduction}

Amyotrophic lateral sclerosis (ALS) is a neurodegenerative disease characterized by progressive weakness and paralysis, caused by motor neuron degeneration and distal motor axonopathy $(1,2)$. In general, ALS is a rapidly progressing disease with survival of 3-5 years after the time of diagnose. The cause of ALS remains largely unknown, and there exists an urgent unmet medical need for drugs that can stop or delay disease progression. In the CNS, ALS is typically accompanied by neuroinflammation involving the emergence of reactive microglia, astrocytes, and aberrant glial phenotypes (3-5). Evidence also shows activation of circulating immune cells (6), as well as immune cell infiltration of the ALS-degenerating peripheral nerves containing motor axons (7-10).

Complex, yet not completely understood, mechanisms underlie the progressive retraction of axons from the muscular motor endplates, a key pathological event leading to paralysis in ALS. Most neuromuscular pathological features of familial and sporadic ALS are reproduced in transgenic mice and rats overexpressing the human ALS-linked mutation of the superoxide dismutase-1 (SOD1 ${ }^{\mathrm{G} 93 \mathrm{~A}}$ ) $(11-13)$. It is reported that motor neuron degeneration in mutant SOD1 mice starts peripherally by a distal axonopathy of the motor nerve branches innervating the neuromuscular junctions (NMJs) before overt loss of motor neuron cell bodies $(2,14)$. Altered axonal transport, defective mitochondria function in motor axon terminals, dys- 
functional Schwann cells, and defective stabilization of NMJs have been proposed as potential pathogenic defects triggering the "dying back" of motor axons (15-17). Finally, evidence for such dying back motor neuropathy has also been found in some clinical forms of $\operatorname{ALS}(2,16)$, suggesting a key pathogenic event that could be targeted by therapeutic interventions.

The role of immune cells influencing NMJ alterations in ALS remains unknown. Previous reports have found macrophage infiltration of peripheral nerves and skeletal muscle in ALS patients and transgenic mice $(7,18)$. However, it remains unknown whether other immune cells interact with motor nerve endings and NMJs. Mast cells are hematopoietic-derived immune cells, whose precursors migrate within tissues reaching maturation and differentiation (19). Mast cells are constitutive immune cells in skeletal muscle, being enriched in tendon regions and perimysium - but in low numbers in the endomysium (20). Mast cells likely play a physiological role in muscle repair and remodeling through the release of different trophic and inflammatory factors, as well as vasoactive mediators (21). Mast cells accumulate in the muscle endomysium following injury or primary myopathies $(21,22)$, with a potential of causing direct lysis of myofibers through the release of proteases (23). In ALS patients, an increased number of mast cells have been described in CNS regions of active degeneration (e.g., motor and prefrontal cortex and pyramidal tracts; ref. 24), suggesting their contribution to neuroinflammation. Nonetheless, scarce information exists about the behavior of mast cells in ALS-affected muscles, such as whether they have a potential role modulating NMJ innervation or triggering local chronic inflammation.

Masitinib mesylate is a selective tyrosine kinase inhibitor that mainly targets type III growth factor receptors including c-Kit, CSF-1 receptor (CSF-1R), and platelet-derived growth factor receptors (PDGF-R), as well as Lyn and Fyn kinases (25-27). These receptors synergistically signal proliferation and migration of cancer and hematopoietic cells, including macrophages. By targeting c-Kit, Lyn, and Fyn, masitinib is particularly efficient in controlling the survival, differentiation, and degranulation of mast cells and, thus, modulating the mast cell-induced array of proinflammatory and vasoactive effects $(25,28)$. Accordingly, clinical trials have shown therapeutic effects of masitinib in cases of mastocytosis, a rare disease characterized by abnormal accumulation and activation of mast cells in various tissues and organs (28). Masitinib was also shown to beneficially modulate CNS function in multiple sclerosis (29), stroke (30), and Alzheimer' s disease (31). In addition, a recent phase III clinical trial with masitinib in ALS has shown promising therapeutic effects in a significant group of patients, slowing the deterioration of motor functions and reducing the decline in quality of life (32). However, the mechanisms of action of masitinib remain largely unexplored, considering that the drug acts on multiple organs and cell types expressing type III tyrosine kinase receptors.

The clinical trial of masitinib in ALS was based on a previous study performed in transgenic rats bearing the ALS-linked SOD1 ${ }^{\mathrm{G} 93 \mathrm{~A}}$ mutation, which showed that postparalysis survival of rats can be significantly extended by systemic treatment with masitinib starting up to 7 days after disease onset (27). This result is significant, considering that in this animal model, a fulminant paralysis develops in rats aged 5-6 months, rapidly progressing from abnormal gait to complete loss of motor function in a period of 2-3 weeks (27). The therapeutic effect of masitinib in ALS rats was associated with the inhibition of CSF-1R in microglia and aberrant glial cells that surround degenerating spinal motor neurons $(33,34)$. Because masitinib is a multifaceted drug affecting multiple immune cell types, we hypothesized that the protective effect of masitinib in ALS likely involves other inflammatory cell types such as mast cells and macrophages in addition to glial cells.

Our primary objective was to search for a morphological interaction between degenerating motor nerve endings and mast cells during the development of paralysis in the hind limb muscle extensor digitorum longus (EDL), a fast-fatigable muscle that becomes largely denervated in the SOD $1^{\mathrm{G} 93 \mathrm{~A}}$ rat model (35). We also explored whether the pharmacologic reduction of mast cells, by means of systemic treatment with masitinib, could modulate the rate of NMJ denervation. Here, we report a sharp increase in mast cell number and degranulation infiltrating the EDL muscle - starting after paralysis onset - and the protective effect of masitinib preventing progressive NMJ denervation.

\section{Results}

Endomysial infiltration and degranulation of mast cells correlate with paralysis progression. In healthy skeletal muscle, mast cells are mainly located at the perimysium and epimysium, with only a small fraction in the endomysium (20). In contrast, endomysial mast cells have been reported to accumulate in response to muscle denervation or myopathies $(22,36)$, suggesting that mast cells are part of a broader inflammatory reaction. Because 
there are no previous studies on the behavior of mast cells in ALS-affected muscles, we analyzed the mast cell number and their phenotypic features in longitudinal sections of the SOD ${ }^{\mathrm{G} 93 \mathrm{~A}}$ rat fast-fatigable EDL muscle. The temporal sequence of events was analyzed at the paralysis onset and after 15 days during advanced paralysis stages, and it was compared with nontransgenic (NonTg) age-matched rats. In the NonTg rat muscles, a low density of mast cells resided in the endomysium, typically displaying uniform granularity when stained with toluidine blue. Compared with NonTg EDL muscle, the density of endomysial mast cells increased by 2-fold at paralysis onset ( 7 vs. 14 cells $/ \mathrm{mm}^{2}$ ) and then sharply augmented by 5 -fold in the following 2 weeks of paralysis progression ( 7 vs. 39 cells $/ \mathrm{mm}^{2}$ ) (Figure 1, A-C). During advanced paralysis, endomysial mast cells were grouped and arranged either in line along some interstitial spaces (Figure 1A) or close to neuromuscular elements (see below), typically displaying increased size and with frequent occurrences of explosive degranulation (Figure 1B). The density of degranulating mast cells, a more relevant measure of mast cell activity and influence in the neuromuscular microenvironment, increased by 8-fold in advanced paralysis EDL muscle as compared with NonTg controls (Figure 1C). Additionally, mast cells in EDL muscle displayed strong immunostaining for tryptase, chymase, and the tyrosine kinase receptor c-Kit, with their colocalization being considered as a specific mast cell marker (Figure 1D).

Mast cells cluster together with motor nerve endings, NMJs, and macrophages during paralysis progression. Peripheral sensory and autonomic nerves have been shown to functionally interact with mast cells, being a mechanism underlying neurogenic inflammation $(37,38)$. However, whether motor nerve terminals interact with mast cells during ALS paralysis progression is presently unknown. Therefore, we used mechanically dissociated EDL muscle preparations to analyze whether the sharp increase of mast cells after paralysis onset was spatially associated with the degenerating motor fibers and NMJs. Figure 2A shows comparative fields of motor nerve branches interacting with motor endplates at different stages of paralysis. In NonTg and SOD $1^{\mathrm{G} 93 \mathrm{~A}}$ onset EDL muscles, only a few mast cells were found in close proximity of motor nerve endings and NMJs (Figure 2A). By contrast, in the advanced paralysis stage, tryptase- and chymase-expressing mast cells were found clustering near or in close contact with degenerating motor nerve endings and NMJs (Figure 2, A and B). Quantitative analysis performed in the whole-mounted EDL muscle showed a significant increase in mast cell density in those areas surrounding the denervated motor nerve terminals and NMJs (neuromuscular compartment), as compared with muscle parenchyma lacking NMJs (Figure 2B). In advanced paralysis, mast cells also consistently interacted with macrophages expressing CD11b/Iba1 and CD68 (Figure 2C and Supplemental Figure 1; supplemental material available online with this article; https://doi.org/10.1172/jci. insight.95934DS1). Endomysial $\mathrm{CD}_{11 \mathrm{~b}^{+}}$macrophages also progressively accumulated within degenerating EDL muscle after paralysis onset and localized in close contact with NMJs. In parallel, CSF-1R immunoreactivity augmented near neuromuscular compartments (Supplemental Figure 1).

Postparalysis tyrosine kinase inhibition with masitinib prevents mast cell accumulation into EDL muscle during advanced paralysis. Masitinib has proven to exert therapeutic effects in ALS patients (32) and to prolong postparalysis survival in SOD1 ${ }^{\mathrm{G} 93 \mathrm{~A}}$ rats (27). Because masitinib potently inhibits the stem cell factor/cKit pathway in mast cells, we reasoned that systemic administration of masitinib $(30 \mathrm{mg} / \mathrm{kg} / \mathrm{d})$ during 15 days after paralysis onset could prevent the augmentation of muscular mast cells in symptomatic rats. Moreover, because masitinib is not expected to exert direct neuroprotection on motor nerve terminals, the masitinib-induced mast cell downregulation would serve as evidence for their pathogenic contribution to NMJ denervation. Figure 3A shows representative transversal sections of the EDL muscle in advanced paralysis from SOD ${ }^{\mathrm{G} 93 \mathrm{~A}}$ rats treated with vehicle and with masitinib. Compared with vehicle-treated rats, masitinib almost completely prevented mast cell accumulation and degranulation, the cell numbers being nonsignificantly different than those of rats at the onset of paralysis (Figure 3, A and B). Masitinib treatment also prevented the interstitial infiltration of macrophages assessed by CD11b staining in the EDL muscle during advanced paralysis (Figure 3C). These results are consistent with a synergistic action of masitinib-downregulating inflammatory activity of mast cells and inhibiting CSF-1R in macrophages (27).

Masitinib-induced reduction of mast cells was associated with preserved innervation of motor endplates. Paralysis progression in ALS correlates with anatomical contact between muscular nerve terminals and motor endplates (39, 40). Thus, we have analyzed the effect of masitinib-induced downregulation of mast cells on the NMJ innervation pattern in the EDL muscle. Figure 4 shows representative microscopic fields illustrating the sequence of progressive denervation between paralysis onset and advanced paralysis as compared with NonTg EDL muscles. About $35 \%$ of EDL muscle motor endplates at onset exhibited denervation, as assessed by NMJ occupancy calculated as motor endplates devoid of presynaptic terminals (yellow colocalization in Figure 4A). Fifteen days 

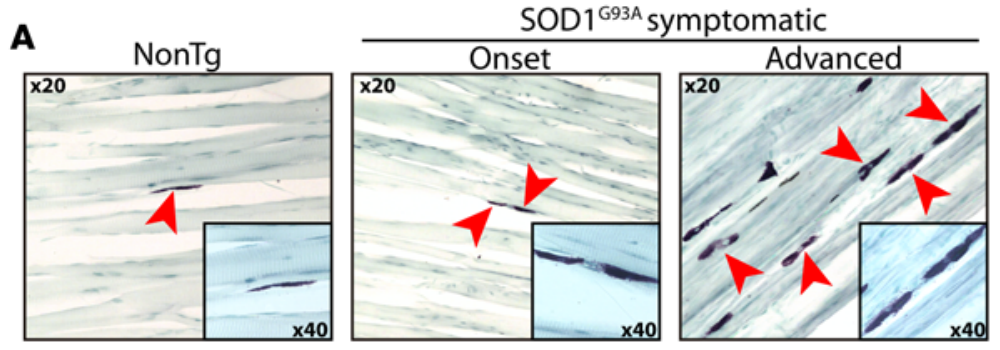

B

SOD $1^{\text {G93A }}$ advanced paralysis

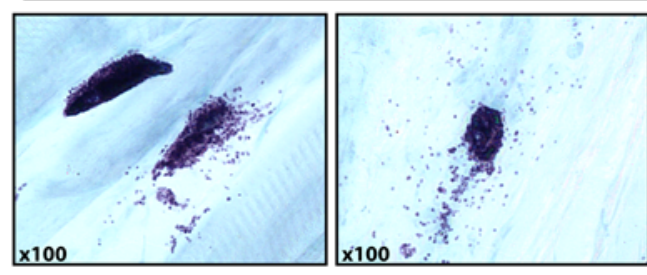

\section{c}

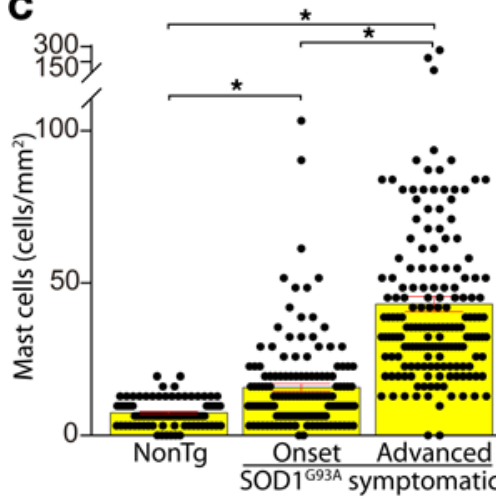

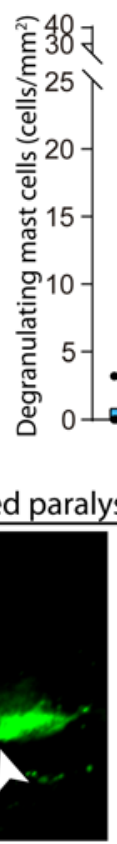
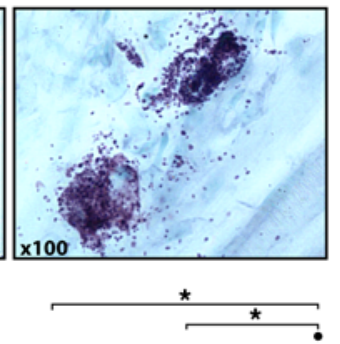
mast cells (arrowheads) from advanced paralysis EDL muscle. Scale bar: $10 \mu \mathrm{m}$. $n=4-5$ animals/condition. stage, displaying the release of metachromatic granules. (C) were analyzed by Kruskal-Wallis followed by Dunn's multiple showing immunoreactivity to tryptase, chymase, and c-Kit in

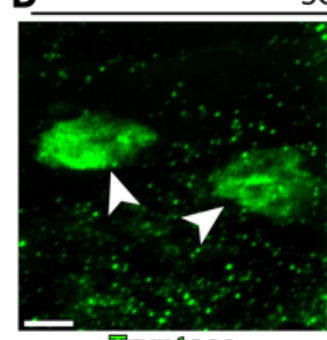

Trypptase
Chymmase

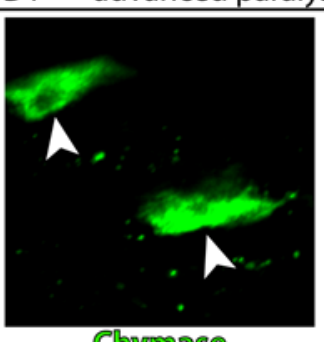

Figure 1. Endomysial infiltration and degranulation of mast cells in the extensor digitorum longus (EDL) muscle of SOD1 ${ }^{\text {c93A }}$ rats. Longitudinal cryostat sections of NonTg and $\mathrm{SOD}^{\mathrm{C} 93 \mathrm{~A}}$ rat (onset and advanced paralysis) EDL muscles were stained with toluidine blue to visualize mast cells. (A) Few endomysial mast cells were observed in NonTg and mSOD1-onset EDL muscles as compared with numerous mast cells during advanced paralysis. Note the sharp increase in mast cell density between onset and advanced paralysis conditions (arrowheads indicate mast cells). Insets show a 40x magnification of Toluidine blue+ mast cells. (B) Representative toluidine blue images of different degranulating mast cell morphologies during the advanced symptomatic Quantitative analysis showing the significant increase in mast cell number and degranulation correlating with paralysis progression. All quantitative data are expressed as mean $\pm \mathrm{SEM}$; data comparison test, ${ }^{*} P<0.01$. (D) Representative confocal images

after paralysis progression, the motor phenotype advanced into advanced paralysis stage and large areas of EDL muscle displayed complete NMJ denervation (data not shown), with the percent of motor endplate denervation being close to $80 \%$ (Figure $4 \mathrm{~B}$ ). Remarkably, masitinib treatment significantly prevented motor endplate denervation with respect to vehicle-treated rats, exhibiting similar denervation values to paralysis onset (Figure 4B). The NMJ protection by masitinib was also evidenced in the functional inverted screen motor test (Figure 4C), where treatment improved the latency-to-fall by $15 \%-25 \%$ from $5-15$ days after onset .

Postparalysis masitinib treatment prevented the pathological remodeling of Schwann cells and vascular networks associated to progressive paralysis. Perisynaptic Schwann cells are glial cells at the NMJs that ensure the synapse stability, maintenance, and repair during damage or denervation (41). Perisynaptic Schwann cells respond to ALS-induced denervation with profound morphological and phenotypic changes (17), retracting processes from the NMJs while extending processes to other endplate areas (42). Therefore, we examined whether downregulation of mast cells in masitinib-treated rats was associated to preservation of perisynaptic Schwann cells in the EDL muscle, as analyzed by confocal microscopy followed by 3-D reconstructions. Figure $5 \mathrm{~A}$ and Supplemental Figure 2 show that NMJ denervation correlated with fewer nestin ${ }^{+}$and $\mathrm{S} 100 \beta^{+}$perisynaptic Schwann cells overlapping with NMJs as compared with those in NonTg or paralysis-onset EDL muscles. In comparison, the number of perisynaptic Schwann cells overlapping with NMJs was largely preserved in masitinib-treated rats (Figure $5 \mathrm{~A}$ ), being comparable with the number at the onset of paralysis. 

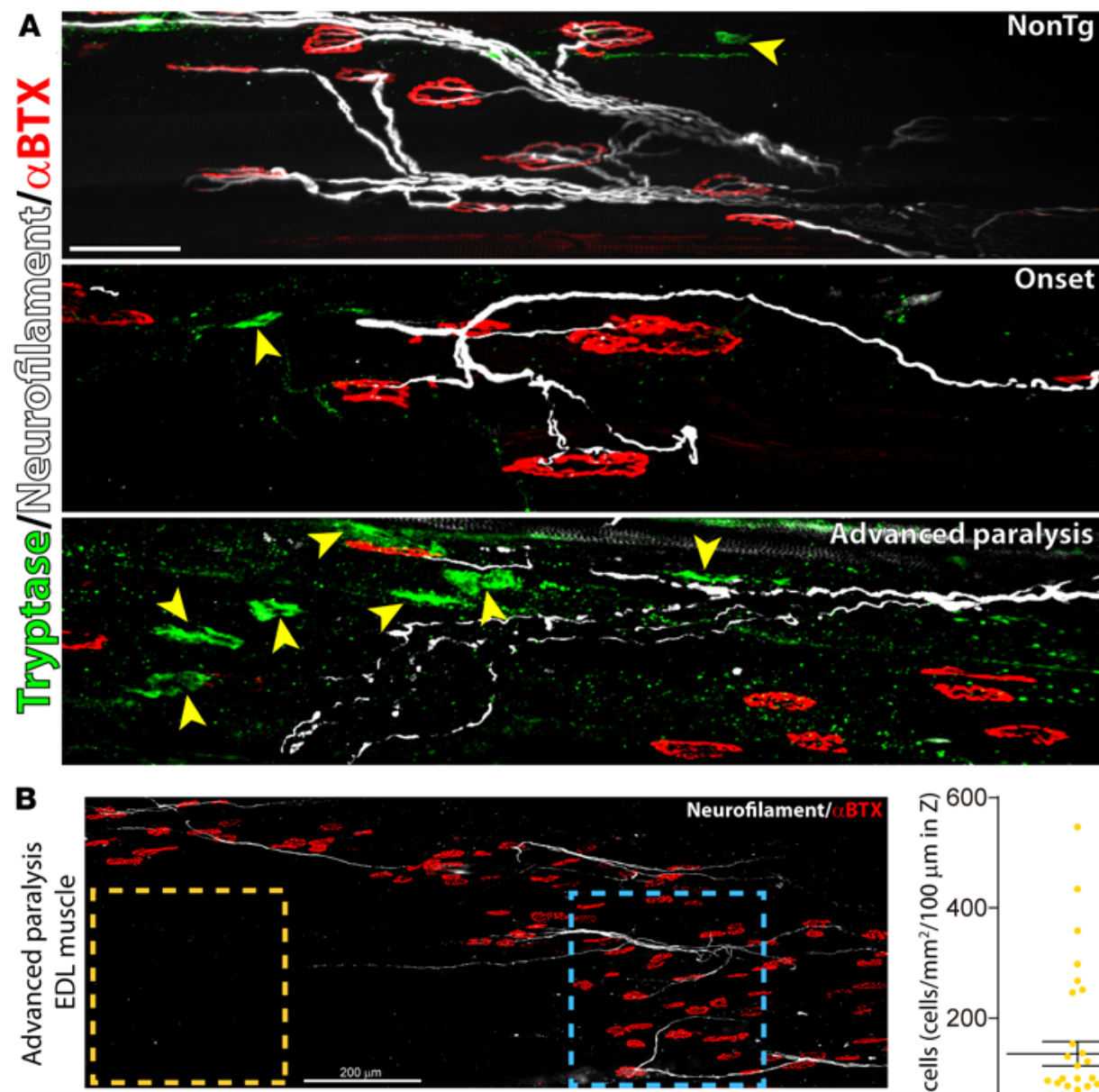

C SOD $1^{\text {G93A }}$ advanced paralysis

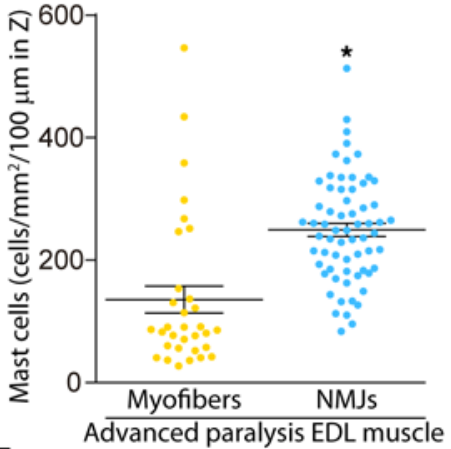

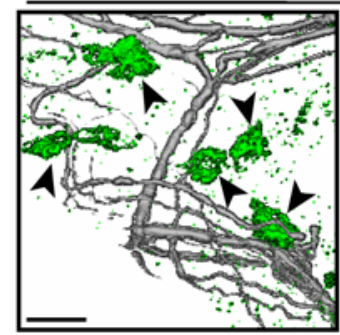

Try/ptase/NF
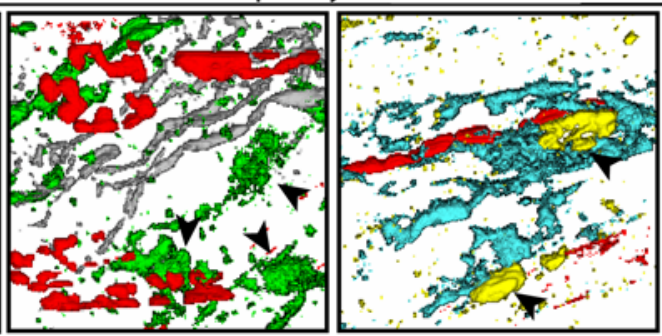

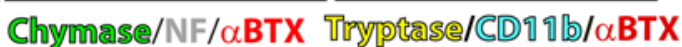

Figure 2. Spatial interaction of mast cells with motor nerve endings, neuromuscular junctions (NMJs), and macrophages during paralysis progression. Whole mount of extensor digitorum longus (EDL) muscles were processed for IHC and visualized in the confocal microscope. (A) Representative confocal images showing the interaction of mast cells with motor nerve endings and NMJs. Branches of motor axons were immunostained for anti-neurofilament (NF, white), motor endplates for $\alpha$-bungarotoxin ( $\alpha$-BTX, red), and mast cells for tryptase (green, yellow arrowheads). Note the clustering of mast cells surrounding degenerating and fragmenting motor nerve endings and denervated NMJs during advanced paralysis ( $n=4$ animals/condition). (B) Comparative analysis of mast cell density in EDL-muscle NMJ compartment (blue square) versus muscle parenchyma devoid of plates (yellow square). The graph shows the cell density expressed as number of cells per $\mathrm{mm}^{2}$ in a $100-\mu \mathrm{m}$ Z-stack. Data are expressed as mean \pm SEM: data were analyzed by Mann-Whitney $U$ test, 2-tailed, ${ }^{*} P<0.01$. Confocal microphotograph in $\mathbf{B}$ is a representative image of the EDL muscle from a symptomatic SOD1C93A rat to illustrate the regions used for quantitative analysis. (C) Representation of the interaction of chymase ${ }^{+} /$tryptase $^{+}$mast cells (green, arrowheads) with motor nerve endings (gray) and NMJs endplates ( $\alpha$-BTX, red). The right panel shows a representative image of the interaction between CD11 $\mathrm{b}^{+}$macrophage-like cells (blue) with mast cells (yellow) in the surrounding of a NMJ (red). Scale bars: $50 \mu \mathrm{m}$ (A) and $15 \mu \mathrm{m}$ (C). $n=4$ animals/condition for $\mathbf{A}$ and $\mathbf{C}$. 
A
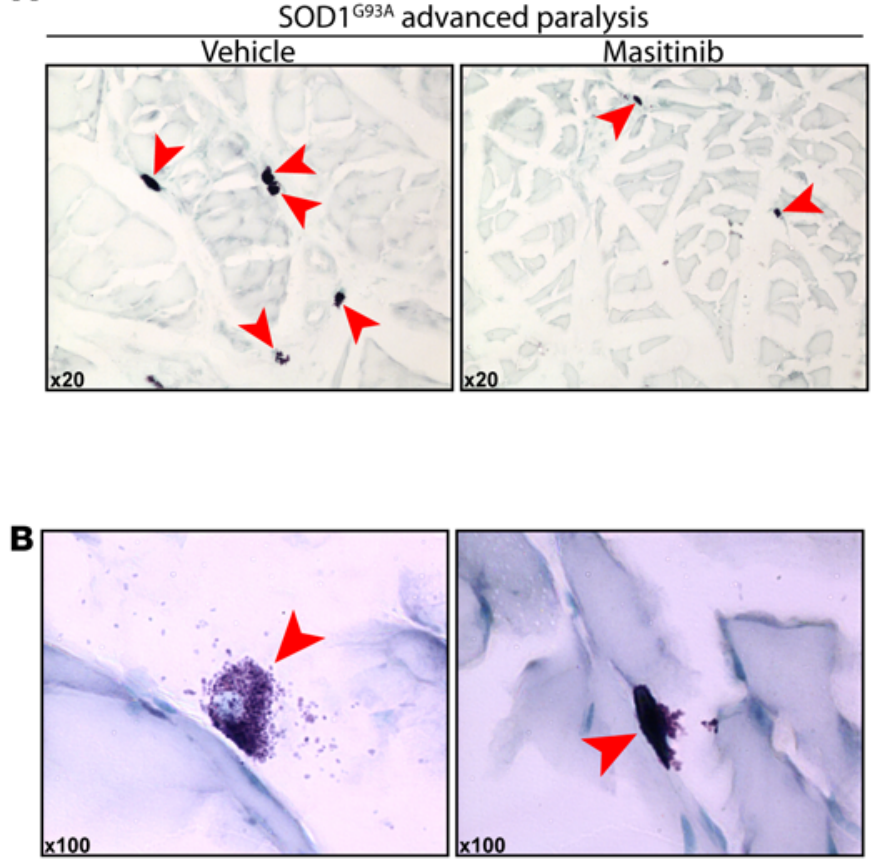

$\times 100$
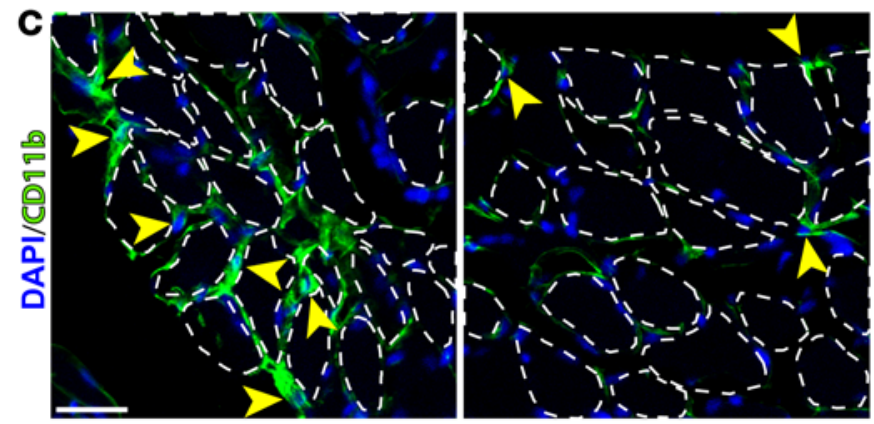

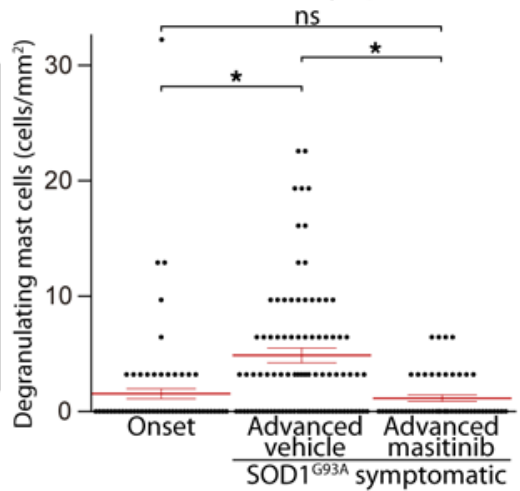

Figure 3. Masitinib treatment reduces mast cell infiltration and degranulation into extensor digitorum longus (EDL) muscle during advanced paralysis. Masitinib $(30 \mathrm{mg} / \mathrm{kg}$ ) or vehicles were orally administered during 15 days after paralysis onset, and rats were processed for histochemical analysis of the EDL muscles. (A and B) Representative microscopic fields of transversal sections of EDL muscle stained with toluidine blue, showing the number and degranulation of mast cells. Note the sharp reduction in mast cell number, size, and degranulating pictures in masitinib-treated rats as compared with controls. The graphs to the right show the quantitative analysis of the number of total mast cells (upper) and degranulating mast cells (lower) assessed in toluidine blue-stained sections. (C) Representative microscopic fields of transversal sections of EDL muscle immunostained with CD11b. Myofibers are delineated by dashed lines. Note the reduction of endomysial macrophage infiltrating the EDL muscles in masitinib-treated rats as compared with controls. The CD11b macrophage-like cell (yellow arrowheads) infiltration area was assessed by IHC. Values for paralysis onset and advanced paralysis-masitinib are expressed as percent respect to advanced paralysis-vehicle. All quantitative data are expressed as mean \pm SEM; data were analyzed by Kruskal-Wallis followed by Dunn's multiple comparison test, ${ }^{*} P<0.01$. $n=4-5$ animals/condition. Scale bar: $25 \mu \mathrm{m}$ (C).

Innervated muscles display a dense network of capillaries that distribute along the interstitial spaces close to myofibrils (43). However, there is scarce knowledge about vascular remodeling occurring in muscles affected in ALS. As shown in Figure 5B, regions with NMJ denervation in EDL muscle 15 days after paralysis onset displayed a systematic reduction of capillary network, suggestive of failing microcirculation. A 15-day treatment with masitinib significantly prevented this pathological vascular remodeling, in parallel with preserved NMJ innervation. 

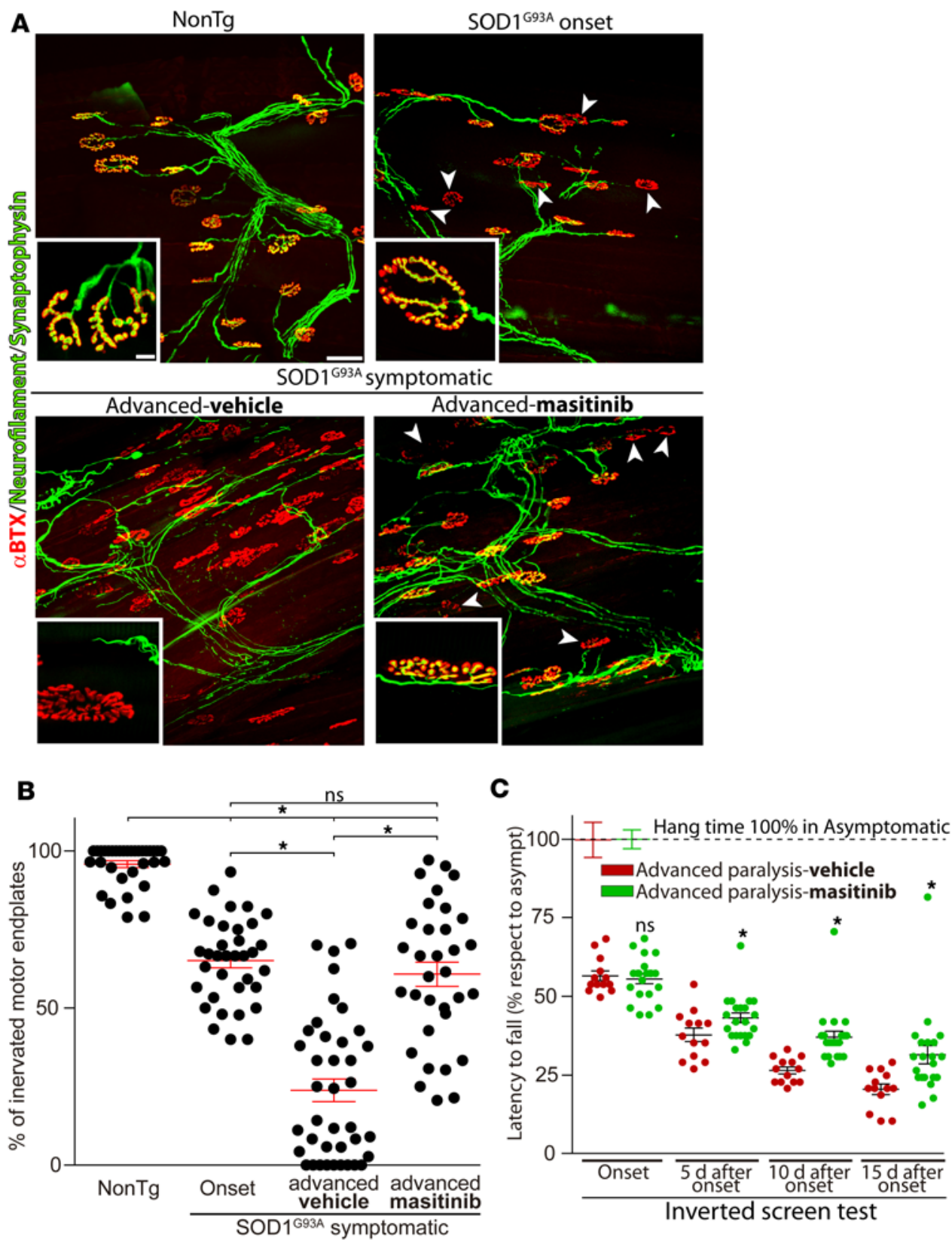

Figure 4. Masitinib treatment prevented neuromuscular junction (NMJ) denervation. (A) Masitinib or vehicle was administered as described above and whole mounted extensor digitorum longus (EDL) muscles were processed for IHC. The panels show representative confocal images used to assess the innervation pattern of NMJs in different experimental conditions. $\alpha$-Bungarotoxin-FITC ( $\alpha$-BTX, red) staining was used to analyze motor endplates. Synaptophysin-Alex Fluor 555 and heavy chain of neurofilaments-Alexa Fluor 555 (green) were used to visualize the motor axon branches and presynaptic terminals. Insets show higher-magnification images of innervated (yellow colocalization) or denervated (red) NMJs. Arrowheads indicate typical denervated motor endplates. Scales bars: $50 \mu \mathrm{m}$ and $10 \mu \mathrm{m}$ for insets. (B) The graph represents the quantitative analysis of NMJ occupancy defined as the overlapping of synaptophysin and $\alpha$-BTX staining and expressed as percentage with respect to the nonTg condition. Note the massive loss of innervation occurring between onset and advanced paralysis in vehicle-treated rats and its prevention by masitinib. $n=4-5$ animals/condition; at least $100 \mathrm{NMJs}$ were analyzed per muscle, per animal. Data are expressed as mean $\pm \mathrm{SEM}$; data were analyzed by Kruskal-Wallis followed by Dunn's multiple comparison test, ${ }^{*} P<0.01$. (C) The inverted screen test was used to measure motor function. For each animal, hang time from an inverted grid was recorded in seconds and expressed as percent of the value of asymptomatic rats (100\%). $n=14$ and $n=22$ for vehicle- and masitinib-treated groups, respectively. Data are expressed as mean \pm SEM; data were analyzed by Mann-Whitney $U$ test, 2 -tailed, ${ }^{*} P<0.01$.

\section{Discussion}

Here, we report that mast cells and macrophages directly interact with degenerating motor nerve endings and motor endplates in symptomatic $\mathrm{SOD} 1{ }^{\mathrm{G} 93 \mathrm{~A}}$ rats. In particular, the increased number and degranulation of mast cells correlated with paralysis progression, suggesting mast cells may be deleterious for the maintenance of functional NMJs. Downregulation of mast cells and macrophage infiltration by means of systemic treatment with the tyrosine kinase inhibitor masitinib significantly reduced the rate of NMJ denervation and motor deficits, further indicating inflammation as a relevant pathogenic mechanism aggravating distal axonopathy and paralysis progression in ALS. Masitinib also prevented the rapid pathological remodeling of Schwann cells and capillary networks observed after paralysis onset, which is consistent with a preserved functioning of the EDL muscle. Remarkably, our findings also provide a significant mechanism of action for masitinib at degenerating NMJs, which may have clinical therapeutic relevance for ALS.

Previous studies have not paid attention to mast cell infiltration in affected muscles from ALS patients or animal models. In symptomatic SOD $1^{\mathrm{G} 93 \mathrm{~A}}$ rats, we found striking changes in the number, phenotype, and spa- 

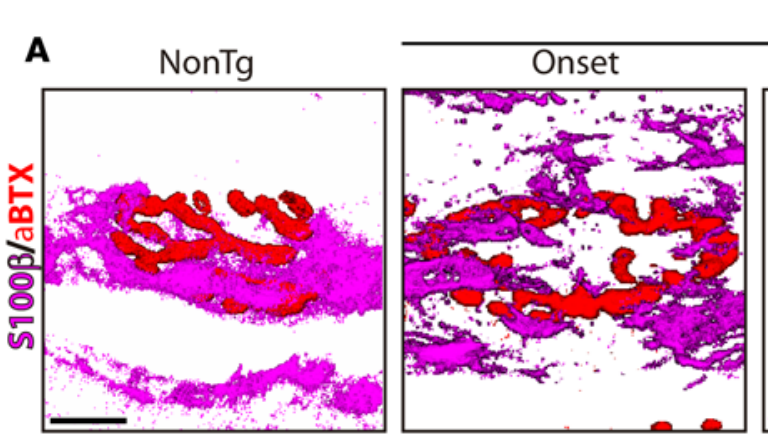

SOD1 ${ }^{\mathrm{G} 93 \mathrm{~A}}$ symptomatic
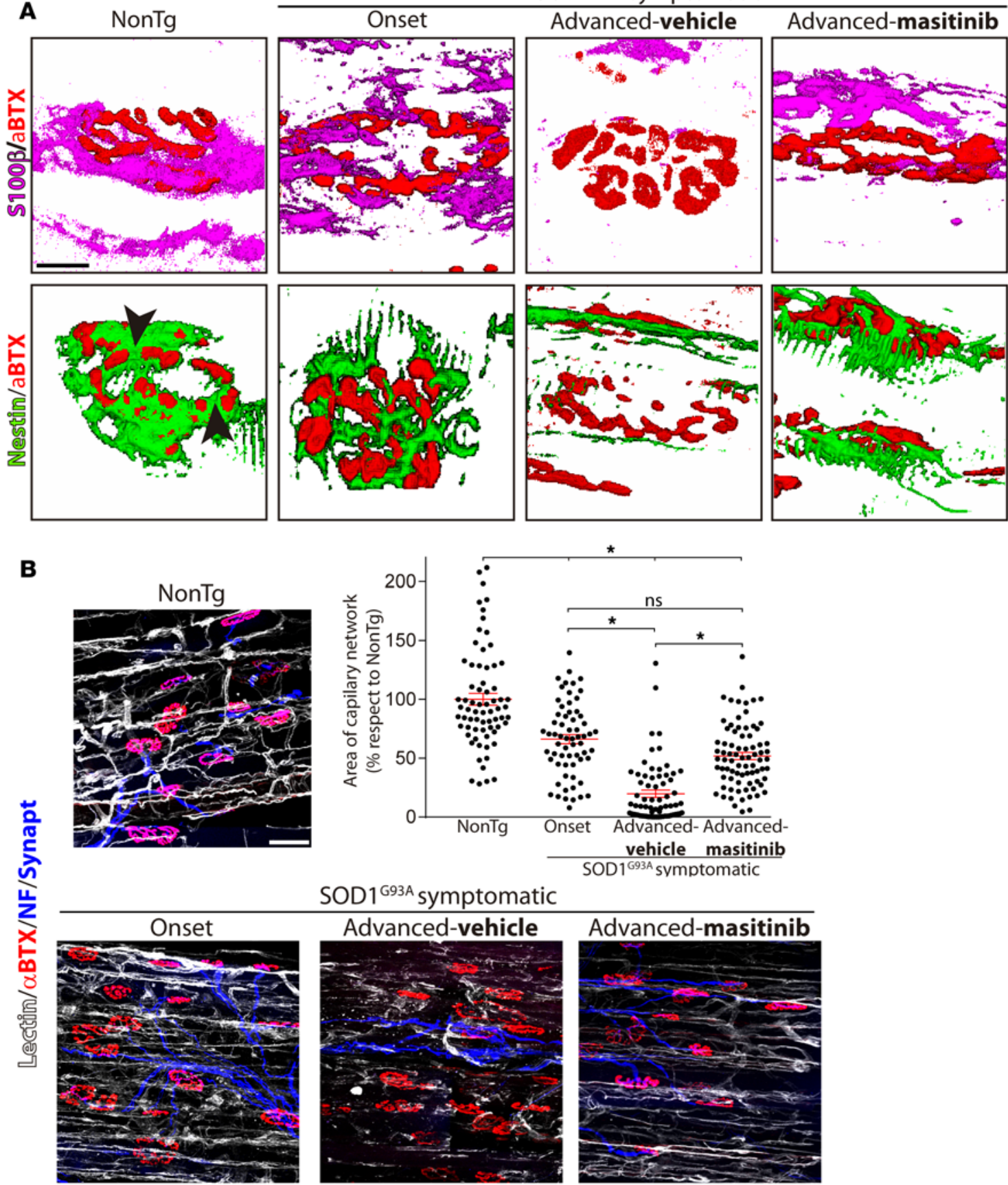

Figure 5. Masitinib prevented perisynaptic Schwann cell and capillary network remodeling in extensor digitorum longus (EDL) muscle during advanced paralysis. Longitudinal cryostat sections of NonTg and SOD1 ${ }^{\text {C93A }}$ rat (onset and advanced paralysis) EDL muscles were processed for IHC to visualize neuromuscular junctions (NMJs), perisynaptic Schwann cells, and the capillary network. (A) Representative 3-D reconstruction of the spatial interactions between $\alpha$-bungarotoxin ${ }^{+}$motor endplates ( $\alpha$-BTX, red) and perisynaptic $5100 \beta^{+}$(magenta) and nestin ${ }^{+}$(green) Schwann cells. Original confocal images are shown in Supplemental Figure 2. In normal NonTg rat EDL muscle, Schwann cells covered the endplates and filled the spaces between adjacent gutters (arrowheads). In contrast, during the symptomatic phase, most nestin ${ }^{+}$and $5100 \beta^{+}$cells progressively retracted from the NMJs no longer overlapping the motor endplates. Masitinib treatment prevented the associated retraction of Schwann cells from NMJs observed during advanced paralysis. (B) Representative images of capillary network assessed with tomato-isolectin staining (white) in EDL muscle areas with high density of motor nerve terminals (blue) and NMJ (red). Note the loss of capillary networks in symptomatic rats. The graph shows the quantitative analysis of total areas stained by lectin in NMJrich regions in different experimental conditions. Data are expressed as mean $\pm \mathrm{SEM}$; data were analyzed by Kruskal-Wallis followed by Dunn's multiple comparison test, ${ }^{*} P<0.01 . n=4$ animals/condition. Scale bars: $50 \mu \mathrm{m}(\mathbf{A})$ and $10 \mu \mathrm{m}(\mathbf{B})$. 
tial location of mast cells in rat EDL muscle, installing in a short period of time (2 weeks) after paralysis onset. These changes are consistent with a causal association between the underlying neuromuscular pathology and a complex inflammatory process, likely orchestrated by degranulation of mast cells, further accelerating denervation. In comparison, mast cell degranulation has been described in patients with muscular dystrophy and autoimmune myositis $(22,44)$, the cells likely inducing injury of myofibers and further recruitment of inflammatory cells through the secretion of proteases $(45,46)$. It is uncertain if similar mast cell-mediated myofiber toxicity also occurs in ALS. Nonetheless, about half of ALS patients exhibit robust increases in serum creatine kinases (47), indicating sustained myofiber injury potentially mediated by mast cell infiltration. Thus, serum creatine kinase might be a useful biomarker of active mast cell-mediated pathology in ALS patients.

The finding of frequent explosive mast cell degranulation in the EDL muscle during advanced paralysis is intriguing, and it anticipate multiple local responses mediated by secreted proteases, cytokines, trophic factors and vasoactive mediators (48). The release of metachromatic granules indicates the secretion of heparin derivatives, which promote regenerative pathways in muscle, mediated in part by FGF signaling (36). At the same time, heparan sulphate may impair the formation of neuromuscular functions in vitro by direct binding to neural agrin (49). The unregulated or overstimulated mast cells can also lead to fibrosis and collagen deposition via the release of several mediators, such as cytokines and TGF $\beta(50,51)$. In turn, fibrosis could adversely affect motor nerve axon plasticity and growth, as well as NMJ reinnervation. A marked fibrotic process in skeletal muscle of symptomatic SOD ${ }^{\mathrm{G} 93 \mathrm{~A}}$ mice has been reported, involving increased deposition of extracellular matrix molecules such as fibronectin and collagen I and -III (52). Mast cells can also release nerve growth factor (NGF) species, leading to the recruitment of T-lymphocytes $(53,54)$ or deleterious signaling through $75-\mathrm{kD}$ neurotrophin receptor $\left(\mathrm{p} 75^{\mathrm{NTR}}\right)$ receptors expressed in either motor nerve terminals or Schwann cells (55). Thus, degranulating mast cells have the potential to directly or indirectly trigger damage of myofibers, motor nerve endings, Schwann cells, and capillary networks.

The proposed pathogenic role of mast cells in the neuromuscular compartment appears to be in conflict with a previous report showing that developmental mutation in kit genes, which limits the expression of c-Kit in SOD1G93A mice, results in reduced survival, despite having a decreased number of mast cells in the spinal cord (56). However, this phenotype could be explained by different concomitant developmental alterations of mice, such as defective myelination and neural progenitors that might create increased vulnerability to mutant SOD1 overexpression. This scenario profoundly differs from our finding of detrimental mast cell activity in ALS, which is spatially and temporally restricted to NMJ pathology during postparalysis stages. Therefore, the appropriate functional phenotype or activation of mast cells appears to be of critical importance, as opposed to their absolute number in the neuromuscular compartment. Consequently, a preferred therapeutic strategy would be downregulation of mast cell activity rather than their pharmacological depletion.

Little is known about the mechanisms of chemoattraction and differentiation of mast cell precursors to ALS-affected muscles. Primary myofiber damage associated with denervation or muscle-restricted expression of mutant SOD1 (57) is a plausible mechanism. Alternatively, a currently unknown mechanism of neurogenic recruitment of mast cells can be anticipated, as evidenced by the close spatial interaction between mast cells and degenerating motor nerve endings and denervated NMJs. A bidirectional interaction between nerves and mast cells is a well-recognized mechanism in neurogenic inflammation triggered by sensory and autonomic nerve endings $(58,59)$. This mechanism is known to be largely mediated by substance $P$ released by axon terminals (60). Substance P does not seem to be expressed in healthy rodent motor neurons but rather is found in significant levels in muscle fibers (61). Other putative mast cell chemoattractant molecules in ALS-affected muscles include TNF $\alpha$, NGF, and ATP produced by either degenerating motor nerve endings, denervated myofibers, perisynaptic Schwann cells, or inflammatory cells. Finally, intact motor nerve terminals release the neurokinin calcitonin gene related protein (CGRP) (62), also capable of modulating mast cell degranulation through specific receptors (58). It is reported that CGRP expression and release in motor neurons is involved in maintenance of NMJs and inversely correlated to neuronal survival (63). Thus, dysfunctional motor nerve terminals might trigger mast cell accumulation and activation in the neuromuscular compartment during ALS.

Mast cell accumulation in EDL muscle was paralleled by accumulation of $\mathrm{CD}_{11} \mathrm{~b}^{+}$macrophage-like cells in the same neuromuscular compartment, suggesting a crosstalk between these immune cells as part of a complex pathological process. Previous studies have described CD11 ${ }^{+}$and $\mathrm{CD} 8^{+}$macrophage infiltrates close to NMJs in SOD ${ }^{\mathrm{G} 93 \mathrm{~A}}$ rats and mice $(7,64)$, a scenario that was partially reverted by protective interventions in ALS rats (64). Also, CD68 ${ }^{+}$macrophages infiltrating the interstitial space between myofibers have been described in the skeletal muscle of ALS patients (18) 
As expected, we found that tyrosine kinase inhibition with masitinib treatment downregulated the mast cell recruitment in degenerating EDL muscle, which in turn resulted in a significant and complete interruption of NMJ denervation in the 15 days after paralysis onset. This mechanism of action of masitinib in muscle was unknown and complementary to a previously described effect of the drug-inhibiting neuroinflammation in the lumbar spinal cord of SOD1 ${ }^{\mathrm{G} 93 \mathrm{~A}}$ rats (27). Thus, the significant increase in SOD1G93A rats after paralysis survival, when treated with masitinib at paralysis onset, might be explained by a simultaneous and probably synergist effect of the drug on deleterious inflammation that accelerates paralysis progression.

Neuroprotection by masitinib was originally associated to inhibition of tyrosine kinase receptor CSF$1 \mathrm{R}$ in microglia and aberrant glial cells that typically proliferate after paralysis onset (27). Because masitinib inhibits various tyrosine kinases, including c-Kit, CSF-1R, PDGF-R, Lyn, and Fyn, it could potentially downregulate other cell types, including mast cells as well as monocytes and macrophages $(25,26)$. In particular, c-Kit receptor activation by stem cell factor is needed for the differentiation of mast cells from precursors (65). It is presently unknown whether stem cell factor is upregulated in ALS-affected muscles; however, it could be expressed by Schwann cells, as described previously in neurofibromatosis type-1 (66).

Masitinib treatment also prevented the regressive remodeling of perisynaptic Schwann cells described in ALS $(67,68)$. Because Schwann cell dissociation from NMJ is mainly caused by the axon retraction from motor plates $(17,68)$, our results further suggest the halt of distal motor axon degeneration following treatment with masitinib. A potential important finding for ALS pathogenesis is the almost complete disappearance we found of capillaries in EDL muscle areas devoid of NMJ innervation. This loss was prevented by masitinib. Degranulating mast cells could potentially mediate this effect through yet-unknown mechanisms. CNS microvascular pathology has been described in ALS (69) and could lead to local hypoxia further promoting recruitment of mast cells, as has been reported in ischemia-reperfusion of skeletal muscle (70). A similar microcirculation failure has been reported in other neuromuscular pathologies such as spinal muscle atrophy or muscular dystrophy $(71,72)$.

In conclusion, the present study addressed one key issue about ALS pathogenesis and therapeutics: the identification of mast cells orchestrating inflammation in the cellular microenvironment of the neuromuscular compartment with the potential to accelerate NMJ denervation after paralysis onset. Mast cells were abundant in the endomysium during paralysis progression and typically clustered with macrophages close to motor nerve terminals, suggesting a neurogenic and/or myogenic induction. Moreover, pharmacological downregulation of mast cells and other immune effectors with masitinib prevented not only NMJ denervation, but also regressive changes in Schwann cells and capillary networks. These mechanisms of action make masitinib a potentially novel ALS drug, capable of exerting multifaceted neuroprotection in both central and peripheral nervous systems via selective kinase inhibition.

\section{Methods}

Animals. Male SOD1 ${ }^{\mathrm{G} 93 \mathrm{~A}}$ progeny were used for further breeding to maintain the line (Taconic Biosciences Inc., NTac:SD-Tg(SOD1G93A)L26H). Rats were housed in a centralized animal facility with a 12-hour light-dark cycle with ad libitum access to food and water. Perfusion with fixative was performed under $90 \%$ ketamine (Phs Pharmaservice) and 10\% xylazine (Xylased) anesthesia, and all efforts were made to minimize animal suffering, discomfort or stress.

Experimental conditions. At least 4 rats were analyzed for each experiment. Four different conditions were studied as follows: i) NonTg rats 160-180 days old; ii) transgenic SOD1 ${ }^{\mathrm{G} 93 \mathrm{~A}}$ rats $180-190$ days old (symptomatic, onset); iii) transgenic SOD $1^{\mathrm{G} 93 \mathrm{~A}}$ rats $195-210$ days old treated with vehicle (symptomatic, advanced paralysis-vehicle), and iv) transgenic SOD ${ }^{\mathrm{G} 93 \mathrm{~A}}$ rats $195-210$ days old treated with $30 \mathrm{mg} / \mathrm{kg} / \mathrm{d}$ of masitinib during 15 days (symptomatic, advanced paralysis-masitinib).

Determination of disease onset and end-stage. As described previously (27), all rats were weighed and evaluated for motor activity daily. Disease onset was determined for each animal when pronounced muscle atrophy was accompanied by abnormal gait, typically expressed as subtle limping or dragging of one hind limb. When necessary, end-stage was defined by a lack of righting reflexes or the inability to reach food and water.

Masitinib administration. As described previously (27), only transgenic rats showing weakness and gait alterations in hind limbs as first clinical sign were selected for masitinib treatment studies. Male and female rats were divided randomly into the masitinib or vehicle-treated groups. Masitinib mesylate (AB1010, manufactured and provided by ABScience), freshly prepared in drinking sterilized water, was administrated daily at a dose of $30 \mathrm{mg} / \mathrm{kg}$ using a curved stainless steel gavage needle with 3-mm ball tip 
(Kent Scientific Corp). Dosing was defined in accordance with previous studies in the same rat model of ALS that was shown to be safe for chronic treatments (27). Rats were treated from day 1 after paralysis for an additional 15 days, when they were then euthanized.

Inverted screen test. Twelve vehicle- and 16 masitinib-treated rats were placed on a metal grid screen $(45 \times 30 \mathrm{~cm})$. After placement, the rats were allowed time to grip before the grid was inverted $50 \mathrm{~cm}$ over a cage containing fresh bedding. Latency to fall was recorded up to 30 seconds. Three independent measurements were recorded 10 minutes apart during the day of testing, and data of 3 measurements were averaged. Measurements started at approximately 5 months ( 1 month before onset, approximately) and continued during 15 days after onset. Latency to fall was plotted (percentage with respect to asymptomatic stage), considering the time of grip during the asymptomatic stage as $100 \%$.

IHC of whole mounted muscle. Rats were deeply anesthetized using 90\% ketamine and 10\% xylazine anesthesia and were fixed by perfusion with 4\% paraformaldehyde (MilliporeSigma) in PBS (AppliChem). Extensor digitorium longus (EDL) muscles from the hind limb were dissected. Then, tissues were blocked for 2 hours at room temperature (5\% BSA, $0.8 \%$ Triton X-100 in PBS), incubated with primary antibodies at $4^{\circ} \mathrm{C}$ overnight: 1:300 mouse monoclonal anti-nestin (GeneTex, GTX26142), 1:400 mouse monoclonal anti-S100 $\beta$ (MilliporeSigma, S2532), 1:250 mouse monoclonal anti-CSF1R (Santa Cruz Biotechnology Inc., sc-46662), 1:300 rabbit polyclonal anti-Iba1 (Wako, 019-19741), 1:250 mouse monoclonal anti-CD68 (Abcam, ab31630), 1:200 mouse monoclonal anti-CD11b (BD Biosciences, BD550299), 1:200 rabbit polyclonal anti-Tryptase (Abcam, ab134932), 1:250 mouse monoclonal anti-chymase (Abcam, ab2377), and rat polyclonal anti-c-Kit-Biotin (Abcam, ab25022). Then, tissue was washed with PBS 3 times for 5 minutes, incubated with secondary antibodies (1:500 goat anti-mouse Alexa Fluor 633 [Thermo Fisher Scientific, A21052], 1:500 goat anti-rabbit Alexa Fluor 546 [Thermo Fisher Scientific, A11035] or -633 [Thermo Fisher Scientific, A21071], 1:500 streptavidin-633 [Thermo Fisher Scientific, \#S21375] and/or fluorescently labeled $\alpha$-bungarotoxin-FITC [ $\alpha$-BTX, Thermo Fisher Scientific, B13422]) for 2 hours at room temperature, washed with PBS 3 times for 15 minutes, and whole mounted in DPX mounting medium (MilliporeSigma) on slides. Axon staining was carried out using 1:1,000 heavy chain neurofilament-Alexa Fluor 555 (MilliporeSigma, MAB5256A5), and axon presynaptic terminals were labeled with 1:300 synaptophysinAlexa Fluor 555 (Abcam, ab206870). Capillary networks were visualized using an isolectin-biotin/ streptavidin-633 1:300 probe (Thermo Fisher Scientific, I21414).

Comparative analysis of mast cells in myofiber and NMJ compartments. Tryptase ${ }^{+}$mast cells were counted in whole-mounted EDL muscle using a stereological approach, contrasting mast cell number in 2 predefined muscle compartments: the region surrounding the motor end plates (NMJ compartment) versus muscle parenchyma devoid of plates. At least 50 confocal stacks $(320 \times 320 \times 100 \mu \mathrm{m}$ in Z-stacks $)$ of 4 SOD1 ${ }^{\mathrm{G} 93 \mathrm{~A}}$ symptomatic animals were analyzed as shown in Figure 2B.

$N M J$ analysis. Structural changes of the NMJ were scored using maximum-intensity projections of images acquired from whole-mounted muscles. Briefly, NMJ innervation analysis was performed taking into consideration those postsynaptic motor endplates occupied by a presynaptic axon terminal, where full innervation was defined as at least $80 \%$ of overlapping between pre- and postsynapsis. An average of 100 NMJs per animal was analyzed using the ImageJ software.

Muscle cryopreserved sections. Paraformaldehyde (4\%) fixed extensor muscle was dissected and cryopreserved in 30\% sucrose (MilliporeSigma) at $4^{\circ} \mathrm{C}$. The 72 -hour preserved muscle was embedded in TissueTek (Sakura) sectioned (longitudinal and transverse) at $10 \mu \mathrm{m}$ using a cryostat and collected on gelatin-coated slides. Sections were blocked for 1 hour at room temperature (5\% BSA, 0.5\% Triton X-100 in PBS), incubated with primary antibodies overnight at $4^{\circ} \mathrm{C}$ (1:250 mouse monoclonal anti-Chymase [Abcam, ab2377], 1:250 rat polyclonal anti-c-Kit-biotin [Abcam, ab25022], 1:200 rabbit polyclonal anti-tryptase [Abcam, ab134932], and 1:200 mouse monoclonal anti-CD11b [BD Bioscience, 550299]), washed with PBS 3 times for 10 minutes, incubated with secondary antibodies for 2 hours at room temperature (1:500 goat anti-rabbit Alexa Fluor 488 [Thermo Fisher Scientific, A11034], 1:500 goat anti-mouse Alexa Fluor 633 [Thermo Fisher Scientific, A21052], and 1:500 streptavidin-Alexa Fluor 633 [Thermo Fisher Scientific, S21375]), washed with PBS 3 times for 5 minutes, and mounted in DPX.

Toluidine blue staining. For the mast cell analysis based in metachromasia observation, $10-\mu \mathrm{m}$ sections of paraformaldehyde-fixed extensor muscle were cryostat sliced and mounted in gelatin-coated slides. Sections were washed and hydrated 2 times in distilled water for 10 minutes and embedded in $1 \%$ toluidine blue solu- 
tion for 10 minutes. Then, slides were washed in distilled water 3 times for 5 minutes and dehydrated during 3 minutes in 70\% ethanol, 3 minutes in 95\% ethanol, and finally 2 minutes in $100 \%$ ethanol. Slides were cleared in xylene twice for 3 minutes each and finally mounted in DPX (MilliporeSigma). Images (10×, 20×, 40×, and $100 \times$ ) were acquired using an Olympus CX41 microscope connected to a Evolution LC Color camera and using ImagePro Express software for adquicition. The number of toluidine blue ${ }^{+}$metachromatic mast cells was counted using the ImageJ software, in at least 40 fields per muscle per animal using a magnification of $10 \times$. Degranulating mast cells, characterized by extensive metachromatic granules being released by an isolated mast cell, were counted using $20 \times$ magnification in serial pictures of each muscle section.

Fluorescence Imaging. Fluorescence imaging was performed with a laser scanning confocal microscope (Zeiss LSM 880 or Zeiss LSM 800) with either a $25 \times$ (1.2 numerical aperture) objective or $63 \times(1.3$ numerical aperture) oil-immersion objective using Zeiss Zen Black software. Maximum intensity projections of optical sections were created with Zeiss Zen software, as were 3-D reconstructions.

Statistics. Quantitative data were expressed as mean \pm SEM. Two-tailed Mann-Whitney test or Kruskal-Wallis followed by Dunn's multiple comparison test were used for statistical analysis, with $P<0.01$ considered significant. GraphPad Prism 7.03 software was used for statistical analyses.

Study approval. All procedures using laboratory animals were performed in accordance with the national and international guidelines and were approved by the Institutional Animal Committee for animal experimentation. All experimental procedures were approved by the Ethical Committee for the use of Experimental Animals (CEUA) of the Institut Pasteur de Montevideo, Uruguay, and under the current ethical regulations of the Uruguayan Law 18.611 for animal experimentation that follows the Guide for the Care and Use of Laboratory Animals of the National Institutes of Health (National Academies Press, 2011).

\section{Author contributions}

ET, LB, OH, and JSB designed the study. ET, SI, RBN, and VV performed experiments. ET, SI, RBN, ICM, PD, and LB analyzed data. ET, OH, JSB, and LB prepared the manuscript. All authors contributed to discussion of the results and edited and approved the final version.

\section{Acknowledgments}

This work was supported by Institut Pasteur de Montevideo - FOCEM Mercosur (COF 03/11), the Amyotrophic Lateral Sclerosis Association (00482), Department of Defense (AL140108), Agencia Nacional de Investigación e Innovación (ANII), Programa de Desarrollo de las Ciencias Básicas (PEDECIBA), and ECOS-Sud Program (U014S02). Partial funding was received from AB Science. We want to thank Colin Mansfield for his critical comments and helpful suggestions revising the manuscript, the staff from the Transgenic and Experimental Animal Unit from Institut Pasteur de Montevideo, and Pablo Fresia for his advice on statistical analysis. Finally, the authors wish to acknowledge the Confocal Microscopy Facility of the Center for Genome Research and Biocomputing at Oregon State University.

Address correspondence to: Luis Barbeito, Institut Pasteur de Montevideo, Mataojo 2020, Montevideo 11.400, Uruguay. Phone: 00.598.2.522.0910; Email: barbeito@pateur.edu.uy.

1. Rowland LP, Shneider NA. Amyotrophic lateral sclerosis. N Engl J Med. 2001;344(22):1688-1700.

2. Fischer LR, et al. Amyotrophic lateral sclerosis is a distal axonopathy: evidence in mice and man. Exp Neurol. 2004;185(2):232-240.

3. Ilieva H, Polymenidou M, Cleveland DW. Non-cell autonomous toxicity in neurodegenerative disorders: ALS and beyond. $J$ Cell Biol. 2009;187(6):761-772.

4. Philips T, Robberecht W. Neuroinflammation in amyotrophic lateral sclerosis: role of glial activation in motor neuron disease. Lancet Neurol. 2011;10(3):253-263.

5. Trias E, Ibarburu S, Barreto-Núñez R, Barbeito L. Significance of aberrant glial cell phenotypes in pathophysiology of amyotrophic lateral sclerosis. Neurosci Lett. 2017;636:27-31.

6. Butovsky O, et al. Modulating inflammatory monocytes with a unique microRNA gene signature ameliorates murine ALS. $J$ Clin Invest. 2012;122(9):3063-3087.

7. Chiu IM, et al. Activation of innate and humoral immunity in the peripheral nervous system of ALS transgenic mice. Proc Natl Acad Sci USA. 2009;106(49):20960-20965.

8. Graber DJ, Hickey WF, Harris BT. Progressive changes in microglia and macrophages in spinal cord and peripheral nerve in the transgenic rat model of amyotrophic lateral sclerosis. J Neuroinflammation. 2010;7:8.

9. Martínez-Muriana A, et al. CSF1R blockade slows the progression of amyotrophic lateral sclerosis by reducing microgliosis and invasion of macrophages into peripheral nerves. Sci Rep. 2016;6:25663.

10. Nardo G, et al. Immune response in peripheral axons delays disease progression in SOD1(G93A) mice. J Neuroinflammation. 
2016;13(1):261.

11. Rosen DR. Mutations in $\mathrm{Cu} / \mathrm{Zn}$ superoxide dismutase gene are associated with familial amyotrophic lateral sclerosis. Nature. 1993;364(6435):362

12. Gurney ME, et al. Motor neuron degeneration in mice that express a human $\mathrm{Cu}, \mathrm{Zn}$ superoxide dismutase mutation. Science. 1994;264(5166):1772-1775.

13. Howland DS, et al. Focal loss of the glutamate transporter EAAT2 in a transgenic rat model of SOD1 mutant-mediated amyotrophic lateral sclerosis (ALS). Proc Natl Acad Sci USA. 2002;99(3):1604-1609.

14. Kennel PF, Finiels F, Revah F, Mallet J. Neuromuscular function impairment is not caused by motor neurone loss in FALS mice: an electromyographic study. Neuroreport. 1996;7(8):1427-1431.

15. Bilsland LG, Sahai E, Kelly G, Golding M, Greensmith L, Schiavo G. Deficits in axonal transport precede ALS symptoms in vivo. Proc Natl Acad Sci USA. 2010;107(47):20523-20528.

16. Dadon-Nachum M, Melamed E, Offen D. The "dying-back" phenomenon of motor neurons in ALS. JMol Neurosci. 2011;43(3):470-477.

17. Arbour D, Vande Velde C, Robitaille R. New perspectives on amyotrophic lateral sclerosis: the role of glial cells at the neuromuscular junction. J Physiol (Lond). 2017;595(3):647-661.

18. Jensen L, Jørgensen LH, Bech RD, Frandsen U, Schrøder HD. Skeletal Muscle Remodelling as a Function of Disease Progression in Amyotrophic Lateral Sclerosis. Biomed Res Int. 2016;2016:5930621.

19. Frenzel L, Hermine O. Mast cells and inflammation. Joint Bone Spine. 2013;80(2):141-145.

20. Sánchez-Mejorada G, Alonso-deFlorida F. Changes in mast-cell distribution in skeletal muscle after denervation. Muscle Nerve. 1992;15(6):716-719.

21. Gorospe JR, Nishikawa BK, Hoffman EP. Recruitment of mast cells to muscle after mild damage. J Neurol Sci. 1996;135(1):10-17.

22. Yokota M, et al. Roles of mast cells in the pathogenesis of inflammatory myopathy. Arthritis Res Ther. 2014;16(2):R72.

23. Porter JD, et al. A chronic inflammatory response dominates the skeletal muscle molecular signature in dystrophin-deficient mdx mice. Hum Mol Genet. 2002;11(3):263-272.

24. Graves MC, et al. Inflammation in amyotrophic lateral sclerosis spinal cord and brain is mediated by activated macrophages, mast cells and T cells. Amyotroph Lateral Scler Other Motor Neuron Disord. 2004;5(4):213-219.

25. Dubreuil P, et al. Masitinib (AB1010), a potent and selective tyrosine kinase inhibitor targeting KIT. PLoS ONE. 2009;4(9):e7258

26. Anastassiadis T, Deacon SW, Devarajan K, Ma H, Peterson JR. Comprehensive assay of kinase catalytic activity reveals features of kinase inhibitor selectivity. Nat Biotechnol. 2011;29(11):1039-1045.

27. Trias E, et al. Post-paralysis tyrosine kinase inhibition with masitinib abrogates neuroinflammation and slows disease progression in inherited amyotrophic lateral sclerosis. J Neuroinflammation. 2016;13(1):177.

28. Lortholary $\mathrm{O}$, et al. Masitinib for treatment of severely symptomatic indolent systemic mastocytosis: a randomised, placebo-controlled, phase 3 study. Lancet. 2017;389(10069):612-620.

29. Vermersch P, et al. Masitinib treatment in patients with progressive multiple sclerosis: a randomized pilot study. BMC Neurol. 2012;12:36

30. Kocic I, et al. Neuroprotective effect of masitinib in rats with postischemic stroke. Naunyn Schmiedebergs Arch Pharmacol. 2015;388(1):79-86.

31. Piette F, et al. Masitinib as an adjunct therapy for mild-to-moderate Alzheimer's disease: a randomised, placebo-controlled phase 2 trial. Alzheimers Res Ther. 2011;3(2):16.

32. Mora J, Barbeito L, Hermine O. Masitinib as an add-on therapy to riluzole is beneficial in the treatment of amyotrophic lateral sclerosis (ALS) with acceptable tolerability: Results from a randomized controlled phase 3 trial. Video Lectures. http://videolectures.net/encals2017_barbeito_mora_hermine_therapy/. Published July 21, 2017. Accessed September 22, 2017.

33. Trias E, et al. Phenotypic transition of microglia into astrocyte-like cells associated with disease onset in a model of inherited ALS. Front Cell Neurosci. 2013;7:274.

34. Díaz-Amarilla P, et al. Phenotypically aberrant astrocytes that promote motoneuron damage in a model of inherited amyotrophic lateral sclerosis. Proc Natl Acad Sci USA. 2011;108(44):18126-18131.

35. Derave W, Van Den Bosch L, Lemmens G, Eijnde BO, Robberecht W, Hespel P. Skeletal muscle properties in a transgenic mouse model for amyotrophic lateral sclerosis: effects of creatine treatment. Neurobiol Dis. 2003;13(3):264-272.

36. Lefaucheur JP, Gjata B, Sebille A. Factors inducing mast cell accumulation in skeletal muscle. Neuropathol Appl Neurobiol. 1996;22(3):248-255

37. Dines KC, Powell HC. Mast cell interactions with the nervous system: relationship to mechanisms of disease. J Neuropathol Exp Neurol. 1997;56(6):627-640.

38. Forsythe P, Bienenstock J. The mast cell-nerve functional unit: a key component of physiologic and pathophysiologic responses Chem Immunol Allergy. 2012;98:196-221.

39. Murray LM, Talbot K, Gillingwater TH. Review: neuromuscular synaptic vulnerability in motor neurone disease: amyotrophic lateral sclerosis and spinal muscular atrophy. Neuropathol Appl Neurobiol. 2010;36(2):133-156.

40. Fischer LR, Glass JD. Axonal degeneration in motor neuron disease. Neurodegener Dis. 2007;4(6):431-442.

41. Sugiura Y, Lin W. Neuron-glia interactions: the roles of Schwann cells in neuromuscular synapse formation and function. Biosci Rep. 2011;31(5):295-302.

42. Kang H, Tian L, Mikesh M, Lichtman JW, Thompson WJ. Terminal Schwann cells participate in neuromuscular synapse remodeling during reinnervation following nerve injury. J Neurosci. 2014;34(18):6323-6333.

43. Plyley MJ, Sutherland GJ, Groom AC. Geometry of the capillary network in skeletal muscle. Microvasc Res. 1976;11(2):161-173.

44. Gorospe JR, Tharp M, Demitsu T, Hoffman EP. Dystrophin-deficient myofibers are vulnerable to mast cell granule-induced necrosis. Neuromuscul Disord. 1994;4(4):325-333.

45. Abonia JP, et al. Mast cell protease 5 mediates ischemia-reperfusion injury of mouse skeletal muscle. J Immunol. 2005;174(11):7285-7291.

46. Oskarsson B. Myopathy: five new things. Neurology. 2011;76(7 Suppl 2):S14-S19. 
47. Iłzecka J, Stelmasiak Z. Creatine kinase activity in amyotrophic lateral sclerosis patients. Neurol Sci. 2003;24(4):286-287. 48. Krystel-Whittemore M, Dileepan KN, Wood JG. Mast Cell: A Multi-Functional Master Cell. Front Immunol. 2015 ;6:620.

49. Wallace BG. Inhibition of agrin-induced acetylcholine-receptor aggregation by heparin, heparan sulfate, and other polyanions. $J$ Neurosci. 1990;10(11):3576-3582.

50. Lee SB, Kalluri R. Mechanistic connection between inflammation and fibrosis. Kidney Int Suppl. 2010;Suppl(119):S22-26.

51. Levi-Schaffer F, et al. Nerve growth factor and eosinophils in inflamed juvenile conjunctival nevus. Invest Ophthalmol Vis Sci. 2002;43(6):1850-1856.

52. Gonzalez D, Contreras O, Rebolledo DL, Espinoza JP, van Zundert B, Brandan E. ALS skeletal muscle shows enhanced TGF- $\beta$ signaling, fibrosis and induction of fibro/adipogenic progenitor markers. PLoS ONE. 2017;12(5):e0177649.

53. Leon A, et al. Mast cells synthesize, store, and release nerve growth factor. Proc Natl Acad Sci USA. 1994;91(9):3739-3743.

54. Levi-Montalcini R, Skaper SD, Dal Toso R, Petrelli L, Leon A. Nerve growth factor: from neurotrophin to neurokine. Trends Neurosci. 1996;19(11):514-520.

55. Tomita K, et al. The neurotrophin receptor p75NTR in Schwann cells is implicated in remyelination and motor recovery after peripheral nerve injury. Glia. 2007;55(11):1199-1208

56. Staats KA, et al. C-kit is important for SOD1(G93A) mouse survival independent of mast cells. Neuroscience. 2015;301:415-420.

57. Wong M, Martin LJ. Skeletal muscle-restricted expression of human SOD1 causes motor neuron degeneration in transgenic mice. Hum Mol Genet. 2010;19(11):2284-2302.

58. Kleij HP, Bienenstock J. Significance of Conversation between Mast Cells and Nerves. Allergy Asthma Clin Immunol. 2005;1(2):65-80.

59. Skaper SD, Facci L, Giusti P. Mast cells, glia and neuroinflammation: partners in crime? Immunology. 2014;141(3):314-327.

60. White DM. Release of substance P from peripheral sensory nerve terminals. J Peripher Nerv Syst. 1997;2(3):191-201.

61. Gundersen K, Oktedalen O, Fonnum F. Substance P in subdivisions of the sciatic nerve, and in red and white skeletal muscles. Brain Res. 1985;329(1-2):97-103.

62. Ringer C, Weihe E, Schütz B. Calcitonin gene-related peptide expression levels predict motor neuron vulnerability in the superoxide dismutase 1-G93A mouse model of amyotrophic lateral sclerosis. Neurobiol Dis. 2012;45(1):547-554.

63. Sala C, Andreose JS, Fumagalli G, Lømo T. Calcitonin gene-related peptide: possible role in formation and maintenance of neuromuscular junctions. J Neurosci. 1995;15(1 Pt 2):520-528.

64. Van Dyke JM, Smit-Oistad IM, Macrander C, Krakora D, Meyer MG, Suzuki M. Macrophage-mediated inflammation and glial response in the skeletal muscle of a rat model of familial amyotrophic lateral sclerosis (ALS). Exp Neurol. 2016;277:275-282.

65. Ito T, et al. Stem cell factor programs the mast cell activation phenotype. J Immunol. 2012;188(11):5428-5437.

66. Ryan JJ, et al. Role for the stem cell factor/KIT complex in Schwann cell neoplasia and mast cell proliferation associated with neurofibromatosis. J Neurosci Res. 1994;37(3):415-432.

67. Loeffler JP, Picchiarelli G, Dupuis L, Gonzalez De Aguilar JL. The Role of Skeletal Muscle in Amyotrophic Lateral Sclerosis. Brain Pathol. 2016;26(2):227-236.

68. Liu JX, Brännström T, Andersen PM, Pedrosa-Domellöf F. Distinct changes in synaptic protein composition at neuromuscular junctions of extraocular muscles versus limb muscles of ALS donors. PLoS ONE. 2013;8(2):e57473.

69. Garbuzova-Davis S, et al. Amyotrophic lateral sclerosis: a neurovascular disease. Brain Res. 2011;1398:113-125.

70. Bortolotto SK, Morrison WA, Han X, Messina A. Mast cells play a pivotal role in ischaemia reperfusion injury to skeletal muscles. Lab Invest. 2004;84(9):1103-1111.

71. Shababi M, Lorson CL, Rudnik-Schöneborn SS. Spinal muscular atrophy: a motor neuron disorder or a multi-organ disease? J Anat. 2014;224(1):15-28.

72. Ennen JP, Verma M, Asakura A. Vascular-targeted therapies for Duchenne muscular dystrophy. Skelet Muscle. 2013;3(1):9. 